\title{
¿Faltan médicos? ¿Cuestión Cuantitativa o cualitativa?
}

\author{
Moderadores: Antonio Alarcó Hernández (ULL) \\ Felipe Rodríguez de Castro (ULPGC)
}

\section{Introducción a la Mesa Redonda}

La SEDEM ha planteado esta mesa redonda porque la pregunta que está en boca de todos, ¿faltan médicos?, plantea demasiados interrogantes que no quedan aclarados con un sí o con un no. Creemos que si solo se formula la pregunta, ¿faltan médicos?, obtendremos una respuesta equivoca. Escuchar un sí o un no, no quiere decir nada sino se está considerando un contexto determinado.

La SEDEM ha solicitado la colaboración en esta mesa redonda de expertos que provienen de diferentes niveles del sistema sanitario con la pretensión de obtener respuestas desde diferentes perspectivas del sistema.

La SEDEM, preocupada por las situaciones contradictorias que plantea la pregunta inicial, solicita a los invitados a la mesa redonda que, antes de responder a las preguntas concretas que se les formula al final de esta introducción, reflexionen sobre los cuatro puntos que desglosamos a continuación.

\section{La percepción de los agentes sociales:}

Los médicos (individualmente): Tienen una sobrecarga de trabajo, por lo que si aumentara la plantilla se repartiría el trabajo, pero ¿piensan repartir los ingresos?

Les corporaciones profesionales: Tienen, o deberían tener, una visión corporativa de conjunto, pero ¿creen que siendo más serán más fuertes como los sindicatos?

Las Asociaciones Científicas de especialidades: Siempre están pendientes de ampliar su territorio de actuación pero, ¿son conscientes de la necesidad de cooperar entre ellas?

Los empleadores: Tienen dificultades en cubrir las necesidades de los servicios actuales, pero ¿necesitan más médicos o necesitan modificar la estructura y funciones de los servicios?

Los pacientes: sufren las listas de espera y piensan que si hubiera más médicos no necesitarían hacer cola, pero ¿piensan en el coste de aumentar las plantillas?

El financiador: ¿Tiene suficientes recursos para comprar los servicios a un precio que permita a los proveedores de servicios ampliar el gasto del capítulo I (aumento de plantilla y de retribuciones)?

El regulador: ¿Tiene una respuesta coherente para el modelo sanitario que regula?

\section{La realidad cuantitativa:}

La respuesta de la Demografía Médica. Datos comparativos entre países relacionados al PIB y a los indicadores sanitarios (expectativa de vida o tasas de mortalidad).

\section{Les respuestas posibles:}

Es necesario un acuerdo sobre el número de médicos necesarios y explicitar porqué nos podríamos permitir tener más:

¿Cuantos médicos nos hacen falta? Disponemos de cálculos ¿basados en las necesidades de los servicios actuales? ¿para reducir las listas de espera? ¿para incrementar la cartera de servicios? ¿sobre las previsibles disponibilidades presupuestarias?

¿Dónde está el problema?: ¿Hay una falta de producción por parte de las Facultades de Medicina? ¿Hay reservas disponibles de médicos inactivos? ¿Hay emigración de médicos? ¿Hay jubilaciones anticipadas innecesarias?

¿Qué tipo de médicos nos hacen falta? ¿Es necesario todo tipo de médicos o solamente de algunas especialidades? ¿A qué se puede deber las faltas específicas y como se pueden solucionar eficientemente? (Por ejemplo, formar muchos más médicos no garantiza tener suficientes psiquiatras).

\section{El Acuerdo:}

Es necesario poner de acuerdo a los diferentes agentes sociales, tanto en su percepción del problema como en su participación en la solución.

A partir de estas reflexiones, la SEDEM propone a cada uno de los expertos de la Mesa redonda I las siguientes cuestiones, siempre en relación al tema genérico de la mesa: ¿Faltan médicos? ¿Cuestión cuantitativa o cualitativa?

\section{Beatriz González López-Valcárcel}

(Universidad de las Palmas de Gran Canaria)

\section{¿Nos podría explicar, desde el punto de vista de la demografía, la situación actual del país en relación a las preguntas que constituyen el tema de la mesa redonda I?}

Los puntos que plantea para la reflexión son atinados y dan en el clavo. Sólo cuando se hacen las preguntas correctas se pueden obtener respuestas útiles. Efectivamente, hay conflictos de intereses, individuales y colectivos. Médicos, pacientes, empleadores y financiadotes públicos de la sanidad presionan en distintas direcciones. Cada uno tendrá su propia versión del déficit de médicos, o superávit, según su conveniencia y percepción.

Individualmente, los médicos asalariados de la red pública serán en general proclives a que se contrate a nuevos efectivos que les reduzcan la carga de trabajo. Los que tienen práctica privada -ejercicio liberal de la profesión- no verán en general con buenos ojos que aumente el número de médicos de su especialidad, que al fin y al cabo son compe- 
tidores potenciales en su mercado. Las sociedades científicas de las especialidades se mueven en la ambivalencia. Por una parte, quieren tener más miembros para aumentar el poder de los números, su capacidad de presión frente a la Administración, etc., y las posibilidades de ir ganando terreno de nadie en la lucha por nuevas competencias profesionales, al hilo del avance tecnológico. Pero por otra parte, representan a médicos individuales a los que interesa mantener la "escasez". La cooperación entre sociedades es difícil porque hay más elementos de disputa que de cooperación. Sólo cuando se enfrentan a un enemigo común vence la cooperación. Los pacientes quieren tener médicos disponibles para atenderles donde y cuando los necesiten, pero por otra parte esos pacientes son ciudadanos que pagan impuestos y financian así la sanidad, no estando interesados en absoluto en sufrir aumentos de presión fiscal.

Esas ambivalencias y conflictos de intereses, que son lógicos y explícitos en una sociedad democrática, no tienen una única respuesta objetiva o aséptica. Inevitablemente, la respuesta implica juicios de valor. Lo mejor que podemos hacer desde el análisis científico es explicitar esos juicios de valor, es decir, afrontar la pregunta sobre el número necesario de médicos con todos los elementos implicados en la respuesta: datos e hipótesis.

Todos los países del mundo regulan la oferta médica. Los mercados educativo y laboral están imbricados y fuertemente regulados por la Administración, que decide desde el numerus clausus de Medicina hasta el número de médicos especialistas a formar o a contratar. Puesto que "producir" un médico especialista lleva más de una década -es una inversión en capital humano a largo plazotodos los países del mundo se plantean la necesidad de planificar su oferta de médicos con horizontes de medio y largo plazo. Incluso lo hacen países de fuerte signo liberal.

La historia de esa planificación es una historia de fracasos. Se puede aprender de ellos, sin embargo. Hay tres métodos de planificación y ninguno es perfecto, la planificación basada en la "necesidad", la basada en la demanda y la que se hace por comparación o "benchmarking". Todos los métodos requieren definir el nivel "óptimo" de médicos activos, generalmente se determinan los ETC: equivalentes a tiempo completo, bajo condiciones de trabajo "estándar".

Las dificultades para definir el nivel "óptimo" de médicos se agravan en España por la precariedad de la información. El registro de médicos, que el MSC está poniendo en marcha, es una necesidad ineludible. Quiero mencionar otras dos dificultades, la primera es la difusa frontera de competencias profesionales entre especialidades, que cambia con el tiempo y con la tecnología, y la segunda es la futilidad de una planificación aislada, en una región o país, en un mundo abierto a las migraciones internacionales de profesionales sanitarios.

¿Faltan médicos hoy en día en España? Bajo un enfoque de demanda, la respuesta es sí. Hay déficits selectivos en algunas especialidades y zonas. Esta afirmación se basa en el análisis de síntomas de exceso de demanda sobre la oferta: plazas sin cubrir por falta de candidatos; bolsas de trabajo en las páginas web de las sociedades profesionales y publicaciones médicas; altísima "cotización" de determinadas especialidades en el mercado MIR.
En un sistema público en el que las remuneraciones no guardan relación con variables específicas del atractivo del puesto ni de la capacitación del candidato, se cubren primero las plazas más atractivas (grandes hospitales docentes con tecnología punta), y el déficit se acaba precipitando en las zonas aisladas o rurales y en los pequeños hospitales comarcales, que no consiguen cubrir plantillas.

En la mesa redonda, si hay tiempo y oportunidad, presentaré algunos datos específicos sobre el alcance de ese déficit.

Algunas especialidades médicas tradicionales se descapitalizarán en pocos años porque una gran parte de sus médicos tienen hoy en torno a los 55 años y se jubilarán, por tanto, a lo largo de la próxima década. Este efecto "cohorte" se debe a la enorme rapidez con que tuvo lugar la expansión de las "residencias" sanitarias de la seguridad social en los años setenta y primeros ochenta. Otra especialidades más jóvenes no tienen ese tipo de problemas.

El déficit de algunas especialidades responde más al tirón de la demanda por expansión profesional y de renta que a problemas demográficos. Cirugía estética, endocrinología o dermatología son tres ejemplos.

La "necesidad" (técnica, objetivable sólo hasta cierto punto) de médicos cambia cada vez más aceleradamente con las posibilidades que abre la tecnología. Especialidades como radiodiagnóstico o cardiología tienen posibilidades de desarrollo profesional que eran impensables hace un par de décadas.

No hay consenso explícito sobre la "necesidad" de médicos por especialidades en términos de estándares, como ratios poblacionales. Ni en España ni fuera. Pero si nos atenemos al principio del "benchmarking" (miremos al vecino que funciona bien, a ver qué ratio de médicos por habitantes tiene), España ocupa una posición intermedia en el mapa de vecinos europeos desarrollados. En la mesa redonda, si hay tiempo y oportunidad, presentaré algunos datos al respecto.

Las necesidades de médicos -personas físicas- han aumentado en España y segurán aumentando en el futuro próximo por los siguientes motivos: 1) feminización de la profesión; 2) tendencia a reducir las horas semanales de trabajo; 3) fuerte tirón de la demanda privada y de las CCAA con transferencias en 2002, que están en proceso de abrir nuevos hospitales y centros de diagnóstico y tratamiento; 4) fuerza propulsora hacia Europa de los médicos españoles, atraídos por las mejores condiciones de trabajo.

\section{José Ignacio Paz-Bouza}

(Universidad de Salamanca. Conferencia

Nacional de Decanos de Medicina)

\section{a) iHan hecho las facultades de medicina una prospectiva sobre el número de alumnos de nuevo ingreso y de egresados en los róximos veinte años en el caso de que la situación se mantuviera como en el momento presente?}

La Conferencia de Decanos ha realizado un minucioso estudio sobre la situación de los médicos españoles a 10, 20 y 30 años, en el supuesto de que el número de alumnos 
de nueva incorporación se mantuviese como ahora y contemplando las jubilaciones, que por razones de edad se producirían en esos años. No hemos podido valorar el número de homologaciones que se puedan producir ni las nuevas necesidades de los diferentes Sistemas Sanitarios del país.

\section{b) En el caso de que la Administración decidiera aumentar el número de alumnos de nuevo ingreso en las facultades de medicina, ¿tienen las facultades los recursos necesarios para asegurar una correcta formación?}

$\mathrm{Si}$ se incrementara el número de alumnos, en las Facultades de Medicina, sería necesario incrementar los recursos, humanos y materiales, para mantener la calidad de la formación. Este hipotético incremento debería de estar justificado en las necesidades de atención a la población y siempre debería de tener un límite. No olvidemos que somos uno de los países de la Comunidad Europea que posee un mayor número de médicos por número de habitantes.

\section{c) ¿Cuál sería el coste (recursos humanos, materiales, sanitarios) de incrementar el número de estudiantes de medicina?}

No es posible hacer una estimación de los costes que se incremento podría suponer, pues dependería, en buena manera, del número del que estuviésemos hablando. Hay que pensar que los grupos, de practicas clínicas, no deben de ser de más de tres alumnos por profesor y en el caso del rotatorio la ratio debería ser un profesor / un alumno. Tendríamos que añadir a esto que se necesitarían más camas hospitalarias. Centros de salud, laboratorios de habilidades clínicas y de comunicación, medios para una correcta evaluación y para el control de la calidad. Esto sería pensando que el incremento lo pudieran asumir las actuales Facultades, si se pensara en nuevas Facultades, como se nos anuncia, habría que añadir los costes que supondrían todos los recursos humanos y materiales de los que habría que dotar e estas Facultades y que por supuesto las otras ya tendrían.

\section{Fernando Pérez Iglesias}

(Asociación de Redes Docentes y Asesoras, AREDA)

\section{a) ¿Desde el punto de vista de la formación médica especializada, faltan médicos en todas las especializadas o las necesidades son específicas de algunas de ellas?}

El dispositivo de formación sanitaria especializada no desarrolla una actividad específica para evaluar adecuadamente la relación entre el número de médicos especialistas existentes en nuestro país y la necesidad de profesionales para cubrir las necesidades asistenciales. Tampoco recibe información sistemática, por parte de las autoridades sanitarias, sobre las necesidades de formación de especialistas y por tanto no tenemos capacidad propia para señalar con certeza el posible déficit de espe- cialistas. Pero si bien no se nos informa ni se nos consulta nuestra opinión sobre el tema, lo que si hacemos desde las Comisiones de Docencia es informar, en cada primavera, la propuesta anual de plazas que el Ministerio de Sanidad propone para la convocatoria del año próximo. Información que va en la línea de asegurar que en los distintos servicios acreditados se mantienen las condiciones necesarias para garantizar la docencia para el período formativo siguiente. No obstante, en el dispositivo de formación especializada, recibimos una cierta información, no oficial, puntual y parcial, sobre plazas vacantes de especialistas, que llega a Jefes de Estudios y Comisiones de Docencia, sobre todo al acercarse la fecha de terminación de una promoción de residentes, en que desde otros hospitales o centros sanitarios se nos pide que informemos, a los que terminan, sobre la existencia de tales vacantes en sus centros. Por tanto, si bien no tenemos elaborada una evaluación sistemática sobre la existencia de déficit de especialistas en nuestro país, y para tenerla debemos recurrir a los escasos estudios realizados sobre el tema, hemos de decir que el dispositivo de formación es quizás un observador privilegiado para evaluar la certeza de los análisis y propuestas de tales estudios. Y en este sentido afirmamos desde el principio, que nos parece evidente que la cuestión de la suficiencia de especialistas es un problema que el Sistema Nacional de Salud debiera estudiar en profundidad y seguir en el tiempo, con el fin, entre otros objetivos, de ordenar la convocatoria de plazas de residentes y la acreditación de centros y unidades docentes.

Los estudios de planificación sobre las necesidades de personal sanitario, tienen una tradición internacional de más de medio siglo, con un amplio desarrollo en países del ámbito de la antigua Unión Soviética y en algunos anglosajones, si bien hay que decir que tales estudios estuvieron jalonados de fracasos por estimaciones erróneas y fueron muy denostados por los economistas del mercado. Nuestro país no tiene mucha tradición en la planificación de las necesidades de especialistas. El primer estudio que conocemos lo realizó la Secretaría General Técnica del Ministerio de Sanidad ${ }^{1}$ en 1984 y dibujaba un panorama con un exceso de especialistas, proponiendo utilizar la oferta de plazas como un instrumento para controlar el número de especialistas futuro, planteando en consecuencia, una disminución de las plazas en las convocatorias MIR, lo que se aplicó a rajatabla y tuvo como consecuencia una reducción de forma dramática en las convocatorias de plazas, ya escasa por entonces y para los próximos años (ver tabla $n^{0} 1^{2}$, lo que ocasionó no solo una escasez grave de cierto tipo de especialistas durante los siguientes lustros (ORL, anestesistas, etc), sinó que además, con una oferta de plazas MIR en algunos años del orden del $14 \%$ sobre el número de licenciados (1984), y cerradas otras posibilidades accesibles de especialización, contribuyó a crear la denominada bolsa histórica de médicos sin titulo de especialista, ocasionado un grave problema profesional y social que se prolongó hasta el momento actual. 
Tabla $\mathrm{n}^{\circ}$ 1: Estudiantes, licenciados en Medicina, plazas MIR y \% de especialización.

\begin{tabular}{lllll}
\hline Curso & $N^{\circ}$ & $N^{\circ}$ & $\begin{array}{l}\text { Plazas } \\
\text { MIR }\end{array}$ & $\begin{array}{l}\% \\
\text { Especialización }\end{array}$ \\
\hline $1977 / 1978$ & 22.554 & 4.563 & -- & \\
$1978 / 1979$ & 19.960 & 6.484 & -- & \\
$1979 / 1980$ & 11.454 & 7.497 & 2.077 & $28 \%$ \\
$1980 / 1981$ & 10.476 & 7.752 & 2.175 & $28 \%$ \\
$1981 / 1982$ & 7.687 & 8.171 & 2.227 & $27 \%$ \\
$1982 / 1983$ & 6.851 & 10.540 & 1.798 & $17 \%$ \\
$1983 / 1984$ & 6.724 & 10.355 & 1.486 & $14 \%$ \\
$1984 / 1985$ & 6.287 & 7.284 & 1.355 & $19 \%$ \\
$1985 / 1986$ & 6.362 & 7.856 & 1.336 & $27 \%$ \\
$1986 / 1987$ & 6.062 & 7.013 & 1.903 & $34 \%$ \\
$1987 / 1988$ & 5.842 & 6.703 & 2.275 & $54 \%$ \\
$1988 / 1989$ & 5.393 & 5.623 & 3.045 & $68 \%$ \\
$1989 / 1900$ & 5.357 & 5.352 & 3.641 & $74 \%$ \\
$1994 / 1995$ & 4.129 & 3.960 & 4.467 & $112 \%$ \\
$1999 / 2000$ & 4.383 & 4.206 & 4.676 & $111 \%$ \\
\hline
\end{tabular}

Más recientemente han realizado estudios globales de oferta y necesidad de médicos y especialistas la OMC en $1995^{3}$ y sobre todo el sindicato médico CESM, que ha presentado diversos análisis, con un enfoque fundamentalmente laboral, y que partiendo de una primera evaluación en $1999^{4}$, que concluía en dictaminar la existencia de una saturación de médicos en el Sistema Sanitario, con excedentes de especialistas que generaban un desempleo superior al $10 \%$, para 9 especialidades, y encontraba como causa los desequilibrios en la convocatoria de plazas MIR entre distintas especialidades, señalando además una creciente feminización del sector como factor reductor de la jornada de trabajo y la previsión de un número importante y creciente de jubilaciones de especialistas para los próximos años, por lo que anunciaba en consecuencia una futura falta de especialistas para otras 10 especialidades. En la causa de estos desequilibrios, la CESM señalaba al Ministerio de Sanidad, por una manifiesta falta de planificación, y a las Comisiones Nacionales de las distintas especialidades, que "establecen el numero de especialistas en función de criterios distintos a los de una planificación racional de los recursos humanos".

El estudio inicial de CESM ha sido actualizado en años posteriores ${ }^{5,6}$, insistiendo cada vez con más énfasis en la aparición de un deficit de médicos y especialistas a cortomedio plazo. La CESM señala ya un déficit en el momento actual para algunas especialidades, como Anestesia ó Pediatría, pero sobre todo indica un grado de envejecimiento notable del colectivo de especialistas en algunas especialidades quirúrgicas, con tasas superiores al $40 \%$ de especialistas en ejercicio por encima de los 50 años, para Cirugía Cardiovascular (48\%), Cirugía Pediátrica (51\%), Neurocirugía (47\%), además de otras 12 especialidades de todo tipo en donde la tasa de mayores de 50 años supera el $30 \%$, especialidades para una parte de las cuales que se anuncia un probable déficit en un próximo futuro sino se hace planificación racional. Estos planteamientos, pronosticando carencias para algunas especialidades, si bien han sido inicialmente cuestionados por alguna administración sanitaria (Cataluña), han coincidido con previsio- nes similares de varias sociedades científicas ${ }^{7,8}$, y ese estado de opinión ha ido ganando adeptos, contribuyendo a crear un consenso general sobre la necesidad de revisar el planteamiento imperante hasta este momento, que daba por sentada la existencia de una plétora de médicos y especialistas para muchos años, y abogando por realizar un estudio serio sobre las necesidades de especialistas para los próximos años, que oriente la convocatoria MIR y acabe con los desequilibrios existentes.

Como resultado de esta mayor sensibilización al problema, en el año 2004, la Comisión de Recursos Humanos del Sistema Nacional de Salud, acordó promover un estudio sobre las necesidades de especialistas, estudio que encargó elaborar inicialmente a las autonomías, para que, en base al mismo realizaran su propuesta para la convocatoria anual de plazas MIR. Al no haber sido recogido el guante por parte de las autonomías, el Ministerio de Sanidad tomó, de nuevo, la iniciativa del estudio, abriendo un concurso para la adjudicación del mismo, el cual recayó en el Grupo de Investigación en Economía de la Salud de la Universidad de las Palmas, que ya había realizado previamente el estudio de necesidades de especialistas para la Sociedad Española de Cardiología. El estudio ha sido presentado en Marzo de 2007 y se denomina "Oferta y necesidad de médicos especialistas en España (2006-2030)" y es probablemente el estudio más completo realizado en España sobre el tema.

Este reciente estudio, que parte de las fuentes de datos tradicionales existentes (colegios de médicos, INE, datos de autonomías), comienza señalando como primera conclusión las limitaciones de tales fuentes y la necesidad de establecer una base de datos oficial, única y actualizada, con la creación de un registro de especialistas actualizable año a año, propuesta que ya ha dado lugar a la publicación de una resolución de la Dirección General de Recursos Humanos sobre registros de profesionales sani$\operatorname{tarios}^{10}$, habiéndose remitido a las CC.AA. un proyecto de convenio entre el Ministerio de Sanidad y las conserjerías de Sanidad, para la creación del registro de especialistas de cada comunidad autónoma.

El estudio utiliza como metodología la dinámica de sistemas, partiendo de una estimación de efectivos en base al año 2006, e identifica las distintas variables y sus relaciones, determina la demanda futura con varias hipótesis, estableciendo proyecciones para el período 2007-2030. Clasifica las especialidades en base a la estructura estaría, coincidiendo en gran parte con los análisis de la CESM, identificando las mismas especialidades con una tasa alta de envejecimiento y señalando a 5 especialidades que ya son deficitarias en el momento actual: anestesia y reanimación, cirugía general y del aparato digestivo, pediatría, radiodiagnóstico y medicina familiar y comunitaria. Además describe el mismo pronóstico de crecimiento del déficit para los próximos años, en base a los principios de feminización, disminución del tiempo de trabajo por reducción de los horarios derivados de la Ley del estatuto Marco y de las directivas europeas, crecimiento de la población por encima de las previsiones previamente estimadas, aumento del nivel de renta, aparición de nuevas tecnologías, etc. Se estima la evolución del número de 
Situación de la lista de espera quirúrgica en el SNS a 31 diciembre 2006

\begin{tabular}{|c|c|c|c|c|}
\hline Especialidades & Total pacientes en espera estructural & Tasa x 1000 habitantes & Porcentaje $>6$ meses & Tiempo medio de espera (dias) \\
\hline Cirugia Gral y Dig. & 71.137 & 1,84 & 6,93 & 68 \\
\hline Ginecología & 21.916 & 0,57 & 2,04 & 61 \\
\hline Oftalmología & 75.342 & 1,85 & 5,02 & 64 \\
\hline ORL & 26.494 & 0,68 & 5,25 & 66 \\
\hline Traumatología & 95.322 & 2,48 & 12,00 & 83 \\
\hline Urología & 24.043 & 0,62 & 3,54 & 61 \\
\hline Cirug. Cardíaca & 2.041 & 0,05 & 3,68 & 73 \\
\hline Cirug. Vascular & 9.433 & 0,24 & 5,78 & 69 \\
\hline C. Maxilofacial & 4.652 & 0,12 & 6,73 & 76 \\
\hline C. Pediátrica & 9.035 & 0,23 & 3,86 & 72 \\
\hline C. Plástica & 10.558 & 0,27 & 8,22 & 82 \\
\hline C. Torácica & 871 & 0,02 & 13,09 & 88 \\
\hline Neurocirugía & 5.133 & 0,13 & 9,19 & 88 \\
\hline Dermatología & 6.805 & 0,18 & 1,01 & 46 \\
\hline TOTAL & 362.762 & 9,38 & 7,07 & 70 \\
\hline
\end{tabular}

Fuente: Sistema de información de listas de espera del SNS.

especialistas existentes en el mercado para los próximos años, en función de la convocatoria de plazas MIR que se ha seguido en los años pasados, y propugna que a partir del año 2008 la oferta de plazas MIR se oriente a las especialidades más demandadas, estudiando varios escenarios de demanda con crecimiento de $0 \%$. 0,5\% y $1 \%$. En la misma línea propone también un incremento de la acreditación de las unidades docentes de las especialidades más demandadas.

El estudio ha sido criticado por los portavoces de varias Comisiones Nacionales de Especialidades, atribuyéndole un carácter excesivamente teórico, pero ha sido aceptado por otras Comisiones Nacionales como una buena base de partida para planificar la formación de especialistas. Como miembro del dispositivo de formación especializada, he de afirmar que coincido con el estudio en la lista de especialidades deficitarias en el momento actual, ya que, entre las 5 señaladas, están la mayor parte de las notificaciones de plazas vacantes, que periódicamente se remiten a las Comisiones de Docencia, para comunicar a los próximos licenciados.

No obstante, antes de terminar, deseo señalar que de los dos estudios principales revisados, el de la CESM hace sus estimaciones a partir fundamentalmente de la situación del mercado laboral profesional, reconociendo que existe pluriempleo en el sector de especialistas, y el del Grupo de Investigación en Economía de la Salud de la Universidad de las Palmas parte de estimaciones de ratios de especialistas por población, y en comparación internacional, califica la situación de nuestro país como de afectado de un exceso de especialistas, y considera la infraestructura de puestos de especialistas del año 2006 como base de la necesidades, sin entrar ninguno de los estudios en cuestionar si la base estructural de puestos de especialistas existentes es adecuada y suficiente. En mi opinión la suficiencia la debe determinar la capacidad de cubrir las necesidades de la demanda asistencial (suficiencia asistencial) durante la jornada de trabajo ordinaria y no un número abstracto, ni tampoco la constatación de la existencia de paro profesional cuando se sabe que coexiste con pluriempleo.

Por tanto, en mi opinión, debieran introducirse en la valoración de la demanda, variables que midan el grado de adecuada cobertura de la demanda sanitaria, como por ejemplo las listas de espera por especialidades, que en nuestro país, con el dispositivo asistencial vigente, se han considerado excesivas y persistentes (ver cuadro de la Agencia de Calidad del $\mathrm{SNS}^{11}$, lo que ante la demanda social para abordar el problema, ha dado lugar a planes de contingencia de casi todas las administraciones autonómicas, con medidas que se entienden como excepcionales y que utilizan "peonadas", derivación de enfermos del subsistema sanitario público al privado, etc., como reconoce el propio Ministerio de Sanidad en la tabla adjunta que tomamos del estudio publicado por el E. Observatory on $\mathrm{HSP}^{12}$. Esta política aparentemente puntual, que se ha iniciado hace más de un lustro, sigue aplicándose año tras año, sobre todo para especialidades quirúrgicas y de métodos diagnósticos, lo que hace pensar que el listado de especialidades deficitarias es superior al que señalan los estudios citados.

\section{b) En el caso de que la Administración} decidiera aumentar el número de alumnos de nuevo ingreso en las facultades de medicina, ¿hay recursos humanos y materiales suficientes a nivel de la formación médica especializada para asegurar esta formación de forma adecuada?:

La propuesta-resumen ${ }^{13}$ realizada por el Ministerio de Sanidad y Consumo, a partir del estudio encargado al Grupo de Investigación de Economía de la Salud de la Universidad de las Palmas, plantea que más allá del déficit selectivo de profesionales afectando a 5 especialidades, existirá en los próximos años un superávit de licenciados, que permitirá abordar las necesidades que se produzcan por las jubilaciones. No obstante el Ministerio propone un esfuerzo de coordinación para ajustar la oferta docente anual a la demanda, la cual la van a determinar las CC.AA. 
ESTRATEGIAS PARA REDUCIR LAS LISTAS DE ESPERA EN LAS CC.AA.

\begin{tabular}{|c|c|c|c|c|c|c|c|c|}
\hline $\begin{array}{l}\text { Comunidad } \\
\text { Autónoma }\end{array}$ & $\begin{array}{l}\text { Planes de } \\
\text { contingencia }\end{array}$ & Peonadas & $\begin{array}{l}\text { Acuerdos } \\
\text { Proveedores } \\
\text { privados }\end{array}$ & $\begin{array}{l}\text { Equipos } \\
\text { quirúrgicos } \\
\text { móviles }\end{array}$ & $\begin{array}{l}\text { Reclutamiento } \\
\text { adicional }\end{array}$ & $\begin{array}{l}\text { Contrato } \\
\text { Programa } \\
\text { (1) }\end{array}$ & $\begin{array}{l}\text { Fondos } \\
\text { específicos }\end{array}$ & $\begin{array}{l}\text { Tiempo } \\
\text { Demora } \\
\text { máxima }\end{array}$ \\
\hline Anadalucia & SI & $\mathrm{SI}$ & SI & $\mathrm{SI}$ & SI & $\mathrm{SI}$ & $\mathrm{SI}$ & $\mathrm{SI}$ \\
\hline Aragón & $\mathrm{SI}$ & $\mathrm{SI}$ & $\mathrm{SI}$ & & $\mathrm{SI}$ & $\mathrm{SI}$ & & $\mathrm{SI}$ \\
\hline Asturias & $\mathrm{SI}$ & $\mathrm{SI}$ & $\mathrm{SI}$ & $\mathrm{SI}$ & $\mathrm{SI}$ & $\mathrm{SI}$ & & NO \\
\hline I.Baleares & & & $\mathrm{SI}$ & & & NO & & estudio \\
\hline I.Canarias & $\mathrm{SI}$ & $\mathrm{SI}$ & $\mathrm{SI}$ & $\mathrm{SI}$ & $\mathrm{SI}$ & $\mathrm{SI}$ & $\mathrm{SI}$ & $\mathrm{SI}$ \\
\hline Cantabria & $\mathrm{SI}$ & $\mathrm{SI}$ & $\mathrm{SI}$ & & & & & $\mathrm{SI}$ \\
\hline C.L.Mancha & $\mathrm{SI}$ & $\mathrm{SI}$ & $\mathrm{SI}$ & & & $\mathrm{SI}$ & & $\mathrm{SI}$ \\
\hline C.-Léon & $\mathrm{SI}$ & $\mathrm{SI}$ & $\mathrm{SI}$ & & & & & $\mathrm{SI}$ \\
\hline Cataluña & $\mathrm{SI}$ & $\mathrm{SI}$ & $\mathrm{SI}$ & & $\mathrm{SI}$ & $\mathrm{SI}$ & $\mathrm{SI}$ & $\mathrm{SI}$ \\
\hline Valencia & $\mathrm{SI}$ & $\mathrm{SI}$ & $\mathrm{SI}$ & $\mathrm{SI}$ & & NO & & estudio \\
\hline Extremadura & $\mathrm{SI}$ & $\mathrm{SI}$ & $\mathrm{SI}$ & & & $\mathrm{SI}$ & & NO \\
\hline Galicia & & $\mathrm{SI}$ & $\mathrm{SI}$ & & & & & NO \\
\hline Madrid & $\mathrm{SI}$ & $\mathrm{SI}$ & $\mathrm{SI}$ & & $\mathrm{SI}$ & $\mathrm{SI}$ & $\mathrm{SI}$ & NO \\
\hline Murcia & $\mathrm{SI}$ & SI & $\mathrm{SI}$ & & SI & $\mathrm{SI}$ & & estudio \\
\hline Navarra & SI & $\mathrm{SI}$ & $\mathrm{SI}$ & & & & & $\mathrm{SI}$ \\
\hline Pais Vasco & $\mathrm{SI}$ & $\mathrm{SI}$ & $\mathrm{SI}$ & & $\mathrm{SI}$ & $\mathrm{SI}$ & $\mathrm{SI}$ & NO \\
\hline Rioja & $\mathrm{SI}$ & $\mathrm{SI}$ & $\mathrm{SI}$ & & & $\mathrm{SI}$ & $\mathrm{SI}$ & NO \\
\hline
\end{tabular}

(1).El contrato programa contiene incentivos económicos para disminuir listas de espera.

Fuente: Ministerio de Sanidad y Consumo. 2004- European observatory on Health Systems and Policies. 2006

con sus estimaciones de necesidad de especialistas, que propondrán al Ministerio para elaborar de la oferta anual de plazas MIR. El ajuste anual de la convocatoria MIR a la demanda autonómica, va a determinar cambios e incrementos en la acreditación de unidades docentes para las especialidades más demandadas, lo que sin duda va a provocar un aumento del número de plazas acreditadas en numerosas unidades docentes y la acreditación de nuevas unidades docentes.

¿Se puede afirmar que el actual dispositivo docente esta en disposición de ofertar esa mayor demanda de formación para las especialidades más deficitarias? En mi opinión, el dispositivo asistencial del Sistema Nacional de Salud tiene capacidad para absorber y formar un número de residentes superior al que recibe actualmente. Como prueba hemos realizado una estimación de la tasa de servicios acreditados sobre el total de servicios hospitalarios en funcionamiento, en la que se observa que en la mayor parte de las especialidades se encuentran acreditados menos del $50 \%$ de los servicios, tal como se puede apreciar para los servicios de los hospitales del antiguo Insalud (hospitales de diez CC.AA. ${ }^{14}$ con datos de servicios en funcionamiento a Diciembre de 2001. Hemos realizado la comparación solo para alguna de las especialidades, incluyendo precisamente a aquellas para las que el estudio del Ministerio de Sanidad prevé un déficit grave ó moderado de especialistas, en el horizonte del año 2010, para un incremento de la demanda moderado del orden del $0,5 \%$.

\begin{tabular}{lllll}
$\begin{array}{l}\text { Especialidades } \\
\text { territorio } \\
\text { INSALUD 2001 }\end{array}$ & $\begin{array}{l}\text { Servicios } \\
\text { instalados }\end{array}$ & $\begin{array}{l}\text { Plazas MIR } \\
\text { convocadas } \\
2001\end{array}$ & $\begin{array}{l}\text { Servicios } \\
\text { acreditados } \\
2006\end{array}$ & $\begin{array}{l}\text { Tasa de } \\
\text { servicios } \\
\text { acreditados }\end{array}$ \\
\hline $\begin{array}{l}\text { Anestesia } \\
\text { Cirugía General }\end{array}$ & 88 & 119 & 34 & $39 \%$ \\
$\begin{array}{l}\text { Medicina Interna } \\
\text { Neumología }\end{array}$ & 79 & 58 & 42 & $49 \%$ \\
$\begin{array}{l}\text { Obstetricia } \\
\text { y Ginecología }\end{array}$ & 83 & 37 & 51 & $58 \%$ \\
Pediatría & 80 & 86 & 26 & $33 \%$ \\
Urología & 86 & 28 & 40 & $48 \%$ \\
\hline
\end{tabular}

Se puede observar que para las 7 especialidades seleccionadas -las de previsible mayor déficit en unos años-, con una tasa de acreditación menor del $58 \%$, se podría incrementar en casi todas un $100 \%$ el número unidades docentes acreditadas, lo que posiblemente puede redundar en un aumento del número de plazas de MIR entre un $25 \%$ y un $50 \%$ sin gran dificultad, ya que si bien la mayor parte de los servicios ya acreditados corresponde a los servicios de los hospitales más grandes y de mayor capacidad docente, en una gran parte de estos servicios docentes el número de plazas de residentes que convocan esta lejos de la saturación, tal como se puede deducir de una tasa media de 2,2 plazas de residentes-año/servicio, para el conjunto de los servicios analizados, con un rango entre menos de 1 residente-año/servicio para la especialidad urología y 4 residentes-año/servicio para pediatría, para un territorio en el que esta incluida la comunidad de Madrid, la sede más importante cuantitativamente de grandes hospitales docentes. Por otro lado solo con adscribir 1 residente/año a cada uno de los servicios no acreditados en el momento actual, se duplicaría el número de residentes para estas 7 especialidades. Hay que recordar que la Ley de Ordenación de las Profesiones Sanitarias establece que toda la estructura asistencial del sistema sanitario esta a disposición de las necesidades docentes 
para la asistencia especializada según establece en su Art. 12 la citada Ley ${ }^{15}$.

Esta previsión no contempla otras consideraciones, como que una parte de los servicios analizados son servicios básicos que estarán implicados en la nueva política formativa de formación troncal, con la posible interferencia sobre la capacidad de formación de un mayor número de especialistas propios de tales servicios, ó la creciente afluencia de médicos extranjeros hacia nuestro país, pero hay que decir que en este año (2007) estamos en el momento de mayor número de plazas MIR convocadas de toda la historia, por lo que posiblemente, en la medida que se haga planificación y se ajuste la oferta anual a las necesidades asistenciales (y no a la llegada de licenciados), se permitirá estabilizar un dispositivo formativo no necesariamente muy superior al actualmente existente, lo que permite afirmar la suficiencia del sistema de formación para la demanda asistencial prevista de un futuro a medio plazo.

Referencias

1. Nadal J, Ruíz F, Rivera J y Gutierrez R. (1984): Oferta y demanda de médicos en España. Una Primera aproximación. Ministerio de Sanidad. Secretaria General Técnica.

2. González B: El mercado laboral sanitario y sus consecuencias en la formación. Numerus clausus. Fundación BBV: La formación de los profesionales de la salud. Escenarios y factores determinantes. (1997).

3. Lancho de León JL, Perteguer F: Médicos especialistas en España. Consejo General de Colegios Médicos de España, Madrid 1995.

4. Fundación CESM 1999: El número de médicos en España en el próximo siglo y sus reprecusiones laborales.

5. Fundación CESM 2000: El número de médicos en España. Análisis de una situación crítica.

6. Amaya C, García MA: Demografía médica en España. Mirando al futuro. Fundación CESM. 2005.

7. Necesidad y disponibilidad de cardiólogos en España. Situación actual, prospectiva y recomendaciones de política de Recursos Humanos. Grupo de Investigación en Economía de la Salud. Universidad de las Palmas. Marzo 2005.

8. Estudio socioprofesional de la Medicina Interna en España. Prospectiva 2010. Sociedad Española de Medicina Interna 2005.

9. OFERTA Y NECESIDAD DE MÉDICOS ESPECIALISTAS EN ESPAÑA (2006-2030). Beatriz Gozález López-Valcarcel, Patricia Barber Pérez. Grupo de Investigación de Economía de la Salud. Universidad de Las Palmas de GC. Marzo 2007.

10. Resolución 7957 de 27 de Marzo de 2007. BOE de 14 de Abril de 2007.

11. SISLE. SNS. Situación a 31 de diciembre de 2006. Agencia de calidad del Sistema Nacional de Salud.

12. Duran A, Lara JL, van Waveren M: Spain. Health system review. Eupean observatory on Health Systems and Policies. 2006.

13. ESTUDIO SOBRE NECESIDADES DE ESPECIALISTAS. Ministerio de Sanidad y Consumo. 2007.

14. Mapa de recursos de asistencia especializada 2001. INSALUD.

\section{Juan-José Rodríguez Sendín}

(Organización Médica Colegial, OMC)

\section{Consideraciones previas}

\section{La percepción de los agentes sociales:}

Los médicos y sus ingresos: Los ingresos relacionados con la carga de trabajo son escasos y en consecuencia, aunque lógicamente se repartieran, tendrían escasa o nula importancia. Es más, la tendencia es ha realizar cada vez menos trabajo duplicar y desdoblar el que se hace porque el mismo tiene escasa relación con los ingresos.

Las corporaciones profesionales: En la Organización Médica Colegial consideramos que globalmente no hay necesidad de mas médicos y que las necesidades son puntuales en ciertas especialidades o bien en algunas zonas y tareas (por ejemplo urgencias y sustituciones).

Los empleadores: En las condiciones actuales ya hemos comentado que se precisan mas médicos en algunas especialidades y para realzar ciertas funciones. Pero esto podría cambiar si la financiación, gestión de personal y de los servicios se modificara.

Los pacientes: No suele ser frecuente que los pacientes realicen planteamientos que no necesitan o no les comprometen de alguna manera. El paciente no puede plantearse coste alguno porque para el los costes que genera su demanda no existen ni en el papel. Para el paciente solo existe su necesidad y la oferta de servicios-

El financiador: Considero que hay suficientes recursos o capacidad para incrementarlos, pero no parece razonable hacerlo a cambio de nada es decir sin avance en salud, en resultados, en tiempo, en calidad. Las retribuciones deben ligarse a resultados y cantidad de trabajo realizado.

El regulador: El regulador no tiene en absoluto una respuesta coherente para el modelo sanitario que regula. Considero que el modelo de SNS esta agotado y debe sufrir cambios profundos.

\section{La realidad cuantitativa:}

La realidad cuantitativa entre los países mas desarrollados es muy dispar. España en número de médicos por habitante se encuentra en los primero lugares de la lista. Pero esto no es suficiente y puede inducir a error porque los modelos son muy diferentes. Quizás ilustren mas las enormes diferencias entre comunidades autónomas e inclusos entre provincias de una misma comunidad autónoma sin que existan diferencias que justifiquen o expliquen la situación.

\section{Les respuestas posibles:}

¿Cuantos médicos nos hacen falta?: Desconozco la respuesta. Es posible obtener proyecciones, partiendo de la realidad en la que nos encontramos y de una supuesta población a la que atender, que nos permitan aventurar un número de médicos con los que se podría responde de igual manera a necesidades similares. Pero las cosas pueden ajustarse mas si previamente se responden cuestiones previas como por ejemplo ¿en un modelo asistencial igual al que tenemos o en otro diferente? Hasta hace pocos años cualquier medico estaba dispuestos a trabajar mas de las 40 horas semanales, ¿conocemos el tiempo medio 
disponible por medico y como evolucionara en los próximos años? ¿Se incentivará alguna vez por lo que se hace y no exclusivamente por desempeñar el cargo? ¿Se permitirá duplicar voluntariamente las horas de trabajo asistencial sobre población distinta a la habitualmente asignada a cambio de incentivos económicos que correspondan o no? ¿Se retribuirá alguna vez el trabajo extraordinario y el complementario al menos como el trabajo ordinario? Es decir se pagara la hora de guardia al menos a igual precio que la hora ordinaria o en su caso por paciente o proceso atendido -como en los recientes acuerdos en el País Vascoó de cualquier otra manera que relacione intensidad del trabajo e incentivos. ¿Se percibirán incentivos económicos relacionados con cantidad y calidad de los servicios prestados? Y definitivamente ¿a que edad se jubilaran los médicos? Estas cuestiones y algunas mas son las que probablemente determinen las profundas diferencias, en cuanto a demografía médica se refiere, entre países similares al nuestro y en consecuencia también determina la respuesta

¿Dónde está el problema?: ¿Hay una falta de producción por parte de las Facultades de Medicina? ¿Hay reservas disponibles de médicos inactivos? ¿Hay emigración de médicos? ¿Hay jubilaciones anticipadas innecesarias? .Todas las respuestas son afirmativas salvo la primera. Las facultades de medicina pueden incrementar el número de médicos para resolver la situación con un modelo de gestión de personal basado no en cantidad calidad y resultados sino en una plétora médica que permitía grandes bolsas de ineficacia, derroche y pérdidas de energía asistencial y sanitaria. No hay estímulos al trabajo con lo cual se tiende a duplicar tareas y funciones o a multiplicar circuitos asistenciales innecesarios para los pacientes.

¿Qué tipos de médicos nos hacen falta?: Ya se comentó anteriormente que las necesidades de médicos son selectivas de algunas especialidades y zonas geográficas. A las segundas es posible responder haciéndolas mas atractivas para los profesionales. Las especialidades deficitarias formando los especialistas necesarios. La troncalidad y formación en áreas especificas ayudaría a paliar el problema.

\section{El Acuerdo:}

Es necesario poner de acuerdo a los diferentes agentes sociales, tanto en su percepción del problema como en su participación en la solución. Por supuesto que si. A este respecto es preciso añadir que son 3-4 años con el diagnostico de situación realizado y salvo el incremento de estudiantes en las facultades no se han tomado mas medidas.

\section{a) Los Colegios Profesionales disponen del registro del número de médicos en ejercicio, ¿Considera que las organizaciones profesionales están en disposición de valorar las necesidades del número de médicos en ejercicio en España?}

Si se mantuviera estable la situación actual si, pero sin tener en cuenta las consideraciones antes mencionadas creo que es difícil porque la situación de oferta y demanda de profesionales es cambiante.

Se puede conocer los profesionales que tenemos, que especialidad tienen cuando van a jubilarse así como su distribución. A partir de ahí deberíamos preguntarnos ¿Qué podemos hacer con esos médicos?, con los que tenemos, que por cierto no son pocos.

\section{En el caso de que se requieran más médicos, ¿necesitamos más de lo mismo o necesitamos otro tipo de médicos?}

Necesitamos médicos de las especialidades deficitarias y médicos con una formación troncal que le permita circular de una especialidad a otra en caso de necesidad o decisión personal.

\section{Boi Ruiz \\ (Unió Catalana d' Hospitals)}

\section{Como empleadores, ¿disponen del número de médicos que necesitan?}

En Cataluña, la atención sanitaria continúa apostado por un modelo de proximidad física al ciudadano de los centros sanitarios. Esto requiere aumentar el número de profesionales en relación a la demanda asistencial. Está claro que no hay suficientes profesionales y que quienes ejercen -como el resto de trabajadores- aspiran a conciliar su actividad laboral con su espacio personal.

Actualmente, la directiva europea limita la jornada laboral ordinaria a un máximo de 48 horas semanales en cómputo trimestral. Los centros ven con preocupación esta medida ya que repercute en el establecimiento de las guardias médicas y se agrava por el derecho de los facultativos a dejar las guardias a determinada edad.

Por tanto, si no se produce un cambio en el modelo asistencial hay que recurrir a corto plazo a profesionales extranjeros y con efectos a largo plazo al aumento de las plazas universitarias de medicina y enfermería.

Lo que está en juego es la equidad de acceso a la calidad asistencial. Para preservarla es necesario revisar el sistema de salud: promover la colaboración entre organizaciones; simplificar servicios; delegar funciones; y establecer una gestión eficaz primando los resultados obtenidos por encima del actual modelo de mando y control.

Bajo medidas solventes a nivel médico, la limitación del número de profesionales debe tener soluciones locales en cada territorio y organización. La revisión del sistema de salud debe promover la racionalización de los recursos. La revista francesa Science et Avenir describe en su Palmares des Hospitaux que nadie duda en hacer 30 kilómetros para escoger una moqueta.

\section{¿Necesitarían un tipo de médicos diferentes del actual?}

Nuestros profesionales están muy bien preparados y son excelentes profesionales, prueba de ello es que son muy bien recibidos en otros países. Lo que sí debería cambiar con rapidez es el sistema de desarrollo profesional y catalogación ad personam, que debería tener efecto en la retribución y debería ser acorde con la ley de ordenación de las profesiones sanitarias.

El actual sistema no prima suficientemente el mérito en el trabajo. El médico debería ser considerado por lo que es, por lo que hace y por cómo lo hace. Esta consideración 
no sólo debería ser social sino también económica. No promover una discriminación en positivo de los más brillantes con postulados igualitaristas perjudica no sólo a estos profesionales sino a todo el sistema.

Asimismo, debería sistematizarse una acreditación común para todos los profesionales a lo largo de su carrera en cuanto a formación, experiencia, artículos publicados, etc. para que en cualquier organización se les valorara de la misma manera.

Por otro lado, los convenios del sector sanitario han de garantizar un nivel salarial y equiparable al de comunidades y estados de nuestro entorno con un nivel similar de riquezaa) Como empleadores, ¿disponen del número de médicos que necesitan?

\section{Ignacio López Puech (Hospital Universitario de Canarias HUC)}

\section{a) Como empleadores, ;disponen en el mercado del número de médicos que necesita?}

Todos los Centros Sanitarios de esta Comunidad Autónoma, tanto de atención primaria como especializada, nos enfrentamos a un déficit crónico de profesionales sanitarios. Esta situación no es homogénea ni por áreas geográficas ni por especialidades.

La oferta de puestos de trabajo es tan amplia en todo el territorio nacional que se acaba estableciendo una especie de competición para captar los últimos residentes que finalizan su periodo de formación, los ofrecimientos de mejores condiciones laborales están produciendo un evidente proceso inflacionista en el que creo que todos nos vemos perjudicados. Las regiones periféricas partimos con desventaja por nuestra situación geográfica, teniendo que cubrir los puestos básicamente con los profesionales formados en Canarias.

Es evidente que la capacidad de atracción sobre los facultativos difiere dependiendo de la complejidad asistencial del Hospital, la posibilidad de ofrecer acceso a las últimas técnicas o tecnologías, las capacidades docentes e investigadoras del centro y en resumen la disposición para un más completo desarrollo profesional ejercen de polo de atracción sobre los médicos. Estos aspectos, unidos a otras consideraciones familiares y evidentemente sociales hacen que en los Hospitales situados en las islas que podríamos denominar "menores" la escasez de profesionales sea posiblemente la prioridad número uno de sus equipos directivos.
En cuanto a la especialidad, hasta hace unos años las dificultades se circunscribían a aquellas en las que el número de residentes que cada año finalizaba su periodo de formación era claramente insuficiente para cubrir las demandas mínimas de nuestro sistema sanitario (anestesia, cirugía pediátrica, cirugía vascular, radiología, etc.). En este momento la situación se ha generalizado a prácticamente todas las especialidades, obligando en algunos casos a limitar las carteras de servicios de los centros sanitarios.

Evidentemente la solución a estos problemas no se va a producir en un plazo corto de tiempo. La formación de especialistas requiere de al menos 10 u 11 años y las medidas que se adopten no verán sus frutos hasta ese momento. La captación de especialistas en otros países (dentro o fuera de la Unión Europea) tiene una repercusión bastante limitada, y en algunos casos se ha puesto en duda la calidad de la atención prestada, los prolongados plazos de convalidación de las titulaciones tampoco han facilitado esta vía.

Todo parece indicar que se va a tener que producir una reordenación de las competencias asignadas a las distintas categorías de profesionales sanitarios, elevando el dintel de cada una de ellas, y sólo desarrollando las funciones para las que sea imprescindible su preparación técnica.

\section{b) ¿Necesitarían un tipo de médicos diferentes del actual?}

En un gran Hospital, que tiene asignada un área de referencia amplia, se precisa y se tiende a la búsqueda de superespecialistas, pero son igualmente necesarios especialistas de base, con una formación más general de su área especifica, que hagan de primer consultor y sean capaces de resolver un gran porcentaje de la patología que les es propia.

En la formación de los médicos se advierten lagunas en aspectos como calidad, gestión, economía sanitaria, medicina basada en la evidencia, organización y trabajo en equipo. La docencia impartida tanto en las facultades como durante el periodo de residencia, basada en conocimientos independientes de cada una de las asignaturas, choca a veces con la organización de los hospitales, basada cada vez más en Unidades Funcionales multidiciplinares y que hacen del paciente el centro de todo el proceso.

No se deben perder tampoco los conceptos más humanísticos de esta carrera y que son imprescindibles para facilitar la comunicación y el trato con nuestros usuarios. 